\title{
Critical Analysis of Non-Nuclear Electron-Density Maxima and the Maximum Entropy Method
}

\author{
R. Y. de Vries, W. J. Briels, and D. Feil \\ Chemical Physics Laboratory, University of Twente, P.O. Box 217, 7500 AE Enschede, The Netherlands
}

(Received 28 November 1995)

\begin{abstract}
Experimental evidence for the existence of non-nuclear maxima in charge densities is questioned. It is shown that the non-nuclear maxima reported for silicon are artifacts of the maximum entropy method that was used to analyze the x-ray diffraction data. This method can be improved by the use of appropriate prior information. We report systematic tests of the improved method leading to the absence of non-nuclear maxima in Si. Likewise, the non-nuclear maxima reported earlier in beryllium are not substantiated. [S0031-9007(96)01044-7]
\end{abstract}

PACS numbers: $31.15 . E w, 61.10 . Y h$

When Besnainou et al. [1] reported a maximum in the calculated electron density distribution (EDD) of the $\mathrm{Li}-\mathrm{Li}$ bond of $\mathrm{Li}_{2}$, this counterintuitive result caused a considerable stir. Whereas this surprising result could be put down to the inherent limitations of the methods they used, the maxima found with restricted configuration interaction (CI) calculations on $\mathrm{Li}$ and $\mathrm{Na}$ clusters by $\mathrm{Cao}$ et al. [2] seemed quite convincing. Next Bersuker et al. [3] performed a full $\mathrm{CI}$ calculation on $\mathrm{Li}_{2}$, which confirmed that the existence of the non-nuclear maximum in $\mathrm{Li}_{2}$ is not an artifact of the level of quantum chemical calculations, and they offered a theoretical explanation of the origin of the effect. Nevertheless, Edgecombe et al. [4] recently reported that the presence of the non-nuclear maxima in the $\mathrm{Na}$ clusters appears to be a basis-set or method-dependent effect. Unfortunately, no experimental methods exist to confirm or invalidate these theoretical results.

We now turn to crystals. Periodicity allows accurate studies of the EDD by x-ray diffraction. In reality, perturbation of the periodicity by the presence either of a surface or of impurities cause oscillations in the EDD, the so-called Friedel oscillations. In the present paper, however, we refer to non-nuclear maxima with the periodicity of the lattice, such as found by Mei et al. [5] in the bcc lattices of lithium and sodium, using the HartreeFock program CRYSTAL [6]. In 1990 Sakata and Sato [7] analyzed the highly accurate x-ray Pendellösung data on Si measured by Saka and Kato [8] with the maximum entropy method (MEM). They found maxima in the electron density in the Si-Si bond. Recently, non-nuclear maxima were also found in Be by Iversen et al. [9], applying the MEM to structure factors that were measured by Larsen and Hansen [10]. These two findings are the first and only experimental support for the rather elusive non-nuclear maxima. They constitute a considerable challenge to theoretical solid state physics, since the many present wave-function calculations on $\mathrm{Si}$, including our own [11], do not corroborate the result. Since we do not doubt the quality of the primary data in the MEM analysis, we decided to reassess the MEM procedure itself and test its ability to bring to light subtle features in electron density maps. In the course of the work we discovered the essential role of the prior assumptions in the analysis, a role that has not yet received the attention it deserves.

Structure factors, obtained from a single crystal x-ray experiment, are directly related to the EDD by means of a Fourier transform. The problems one is facing when performing the inverse Fourier transformation are that (i) the experiment provides us with a limited number of structure factors, (ii) all structure factors are determined within a certain experimental error, and (iii) the phase of the structure factors is unknown. In case the crystal is centrosymmetric the phases can usually be derived without any ambiguity and only the first two problems remain.

The traditional way to extract the EDD from a limited set of noisy data is to fit the structure factors by a model. The drawback of this method is that features which are not allowed by the model will never show up in the EDD. Furthermore, random errors are traded in for systematic errors [12].

The MEM of Jaynes $[13,14]$ seems indeed to be a more proper method to handle these problems, since no model is presupposed. The MEM was introduced by Gull and Daniell [15] in 1978 for constructing a map from blurred radioastronomical data. Based on arguments of Frieden [16] they concluded that the most probable image that can be extracted from an incomplete and noisy set of data is the one that is consistent with the data and maximizes $S=-\sum_{i} p_{i} \ln p_{i}$ where $p_{i}$ is the normalized intensity at pixel $i$ of the digitized image. The same arguments hold for extracting an EDD from incomplete and noisy Fourier data. The unconstrained maximum of $S$ is the uniform EDD. Maximizing $S$ subject to the constraints given by the experiment this method will give the EDD that is closest to the uniform distribution. It is this method that leads to the erroneous results as will be shown below.

Kullback and Leibler [17] introduced the concept of minimum cross entropy. Instead of striving towards a featureless EDD as the MEM does, this method strives towards close resemblance to a given "prior" distribution. More concretely, the quantity $\sum_{i} p_{i} \ln \left(p_{i} / m_{i}\right)$ is 


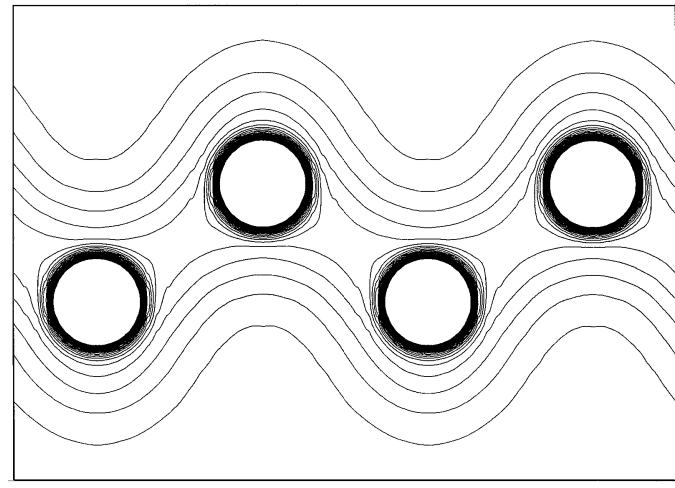

FIG. 1. The EDD of silicon in the 110 plane obtained by a Fourier synthesis of the structure factors for which $\sin (\theta) / \lambda<5.5 \AA^{-1}$. Contour intervals are at $0.1 e / \AA^{3}$ (cutoff level $\left.2.5 e / \AA^{3}\right)$.

minimized where $m_{i}$ is an EDD that is used as reference. We have augmented our computer program TWENTROPY [18], based on the algorithm of Skilling and Bryan [19], in order to allow for a prior distribution. In our case, the prior distribution chosen was the EDD of a "procrystal," which already contains the atomic peaks. The EDD of the procrystal was obtained by calculating the structure factors of the lattice of free atoms, applying the DebyeWaller factor, and transforming back to real space. Recently, a similar analysis has been performed by Zheludev et al. [20] when reconstructing spin densities from incomplete and noisy Fourier data. They concluded that the use of a nonuniform prior distribution greatly enhances the quality of the reconstructed maps.

The most common way to incorporate the experimental data is to maximize the entropy subject to

$$
\chi^{2}=\sum_{h k l} \frac{\left(F_{\mathrm{obs}}-F_{\mathrm{calc}}\right)^{2}}{\sigma_{h k l}^{2}}=M,
$$

where $F_{\text {obs }}$ is the observed structure factor, $F_{\text {calc }}$ is the structure factor calculated from the density, $\sigma_{h k l}$ is the standard deviation, and $M$ is the total number of (unique) reflections.

With co-workers we have recently concluded [11] that the non-nuclear maximum in silicon is probably an artifact of the MEM. We calculated the EDD of silicon with the density functional program ADF-BAND [21]. Structure factors were calculated from this static density, and isotropic thermal vibration was introduced via a Debye-Waller factor $\left(B=0.4642 \AA^{-2}\right.$, taken from [22]) [23]. The resulting EDD, calculated by means of a Fourier transformation of a full set of structure factors, did not show any non-nuclear maximum in the Si-Si bond; see Fig. 1. From these theoretically generated structure factors (the model data) we selected a subset with the same $h, k$, and $l$ values as the set used by Sakata and Sato [7] and applied the MEM to it (on a $128 \times 128 \times$ 128 grid). The theoretically generated structure factors

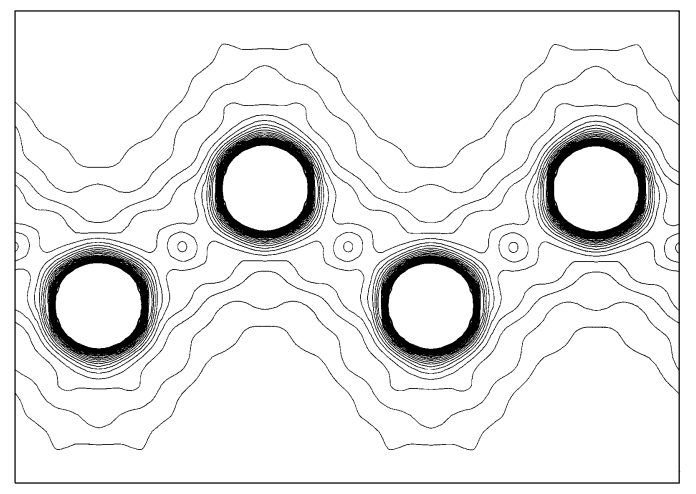

FIG. 2. MEM analysis of model data using the same set of structure factors as were measured by Saka and Kato. Grid $128 \times 128 \times 128$ pixels. Contour intervals are at $0.1 e / \AA^{3}$.

are free of errors and therefore should have $\sigma_{h k l}=0$. However, since this leads to calculational problems we have chosen $\sigma_{h k l}$ very small $\left(\sigma_{h k l}=0.0005\right.$, compared to $\sigma_{h k l} \approx 0.05$ for the experimentally obtained structure factors [8]). In the resulting EDD non-nuclear maxima in the $\mathrm{Si}-\mathrm{Si}$ bond are found that are very similar to the ones obtained by Sakata and Sato [7]; see Fig. 2. Of course, we know in this case that the maxima are erroneous. The atomic peaks in the EDD constitute features that cause a considerable reduction in entropy

(a)

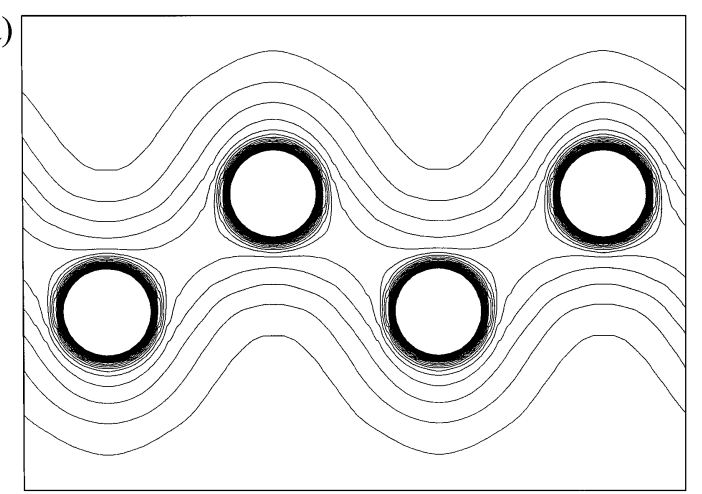

(b)

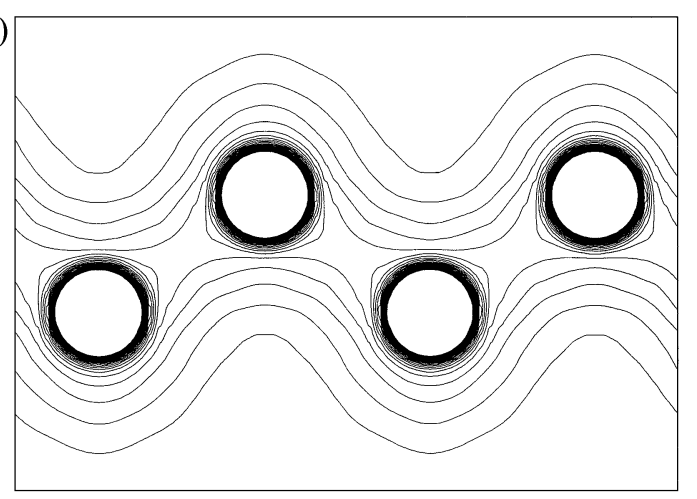

FIG. 3. MEM analysis including the procrystal as a prior distribution on the same set of structure factors that was measured by Saka and Kato. (a) Model data. (b) Experimental data. Contour intervals are at $0.1 e / \AA^{3}$. 
compared with the uniform distribution that is normally used as prior reference in the MEM. The MEM uses the freedom allowed by the undetermined values of the absent structure factors to reduce the height of these peaks. This can lead to false detail in the bonding density region [11].

Applying the MEM to the model data, this time including the procrystal as a prior distribution, leads to an EDD shown in Fig. 3(a). No non-nuclear maxima between two bonded silicon atoms are found. Furthermore, the obtained EDD is very similar to the original EDD (Fig. 1). When the calculation is done using the experimental data of Saka and Kato [8] we find an EDD shown in Fig. 3(b), again, without non-nuclear maxima in the $\mathrm{Si}-\mathrm{Si}$ bond.

The fact that non-nuclear maxima are absent in case the procrystal is used as a prior distribution is no proof of the nonexistence of the non-nuclear maxima. What if the non-nuclear maxima are really there? Will the MEM with a theoretically generated prior distribution be capable of showing them? To test this we have created an EDD of silicon and added charge in the Si-Si bonds (by adding an EDD with a Gaussian shape) to simulate a non-nuclear maximum. The result is shown in Fig. 4(a). Structure factors were calculated from this density, and the same set that was measured by Saka and Kato [8] was used in the analysis. The result is shown in Fig. 4(b). We see that

(a)

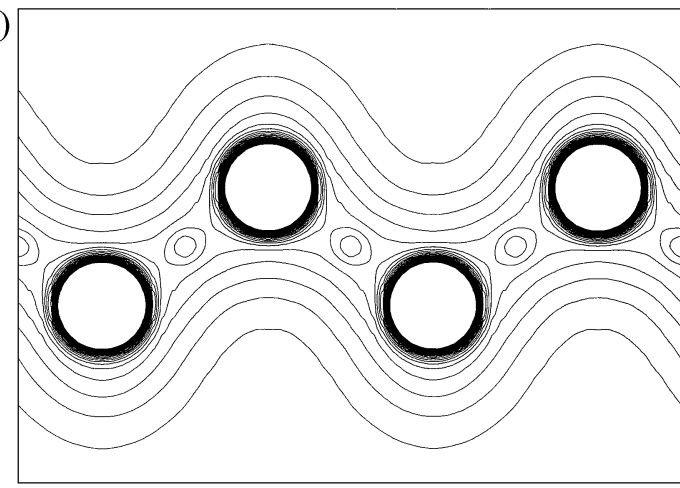

(b)

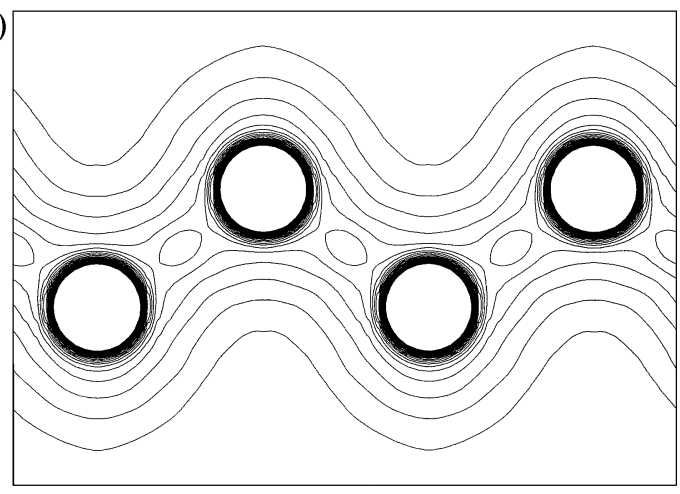

FIG. 4. (a) EDD of silicon with charge added in the Si-Si bond to simulate a non-nuclear maximum. (b) MEM analysis of the data that was calculated from the EDD where charge was added in the $\mathrm{Si}-\mathrm{Si}$ bond to simulate a non-nuclear maximum. The set of data was the same as the one measured by Saka and Kato. Contour intervals are at $0.1 e / \AA^{3}$. non-nuclear maxima are indeed found between $\mathrm{Si}$ atom pairs, although the height of the maxima are lower than the original maxima. This means that if there is evidence in the data for the existence of a non-nuclear maximum in the Si-Si bond, then the MEM with our type of prior distribution should still show this non-nuclear maximum.

From this analysis we conclude that the MEM provides a reliable method to extract EDDs from experimental data, provided the procrystal is used as a prior distribution.

This, together with the theoretical results of Fig. 1, leads us to conclude that there is no non-nuclear maximum in the Si-Si bond.

Subsequently, we turned to Be metal to see if the nonnuclear maxima obtained by Iversen et al. [9] still show up in the EDD of beryllium metal when the procrystal is used as a prior distribution. Again, the procrystal was

(a)

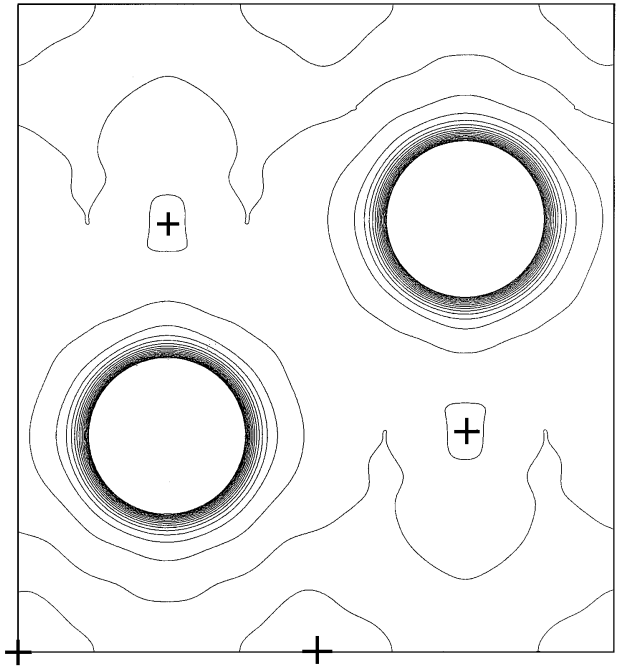

(b)

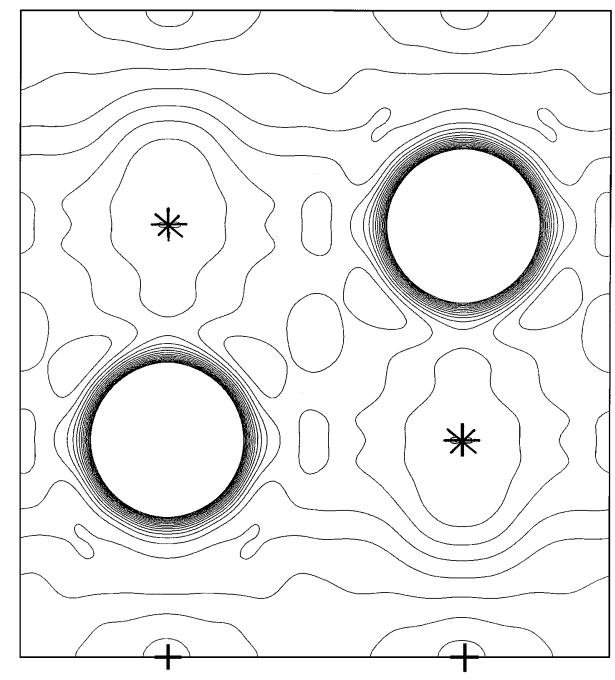

FIG. 5. MEM analysis of the dataset of beryllium measured by Larsen and Hansen. (a) (110) section, the procrystal was used as a prior distribution and (b) with a uniform prior distribution. The points marked with a + are minima, $*$ are maxima. Conour intervals are at $0.025 e / \AA^{3}$ (cutoff level: $\left.0.625 e / \AA^{3}\right)$. 
calculated as a sum of thermally smeared free atoms. The temperature factors were taken from [10]. The result of the MEM analysis using the procrystal as a prior distribution is shown in Fig. 5(a) (grid $120 \times 120 \times$ 120). No non-nuclear maxima are found in the EDD of beryllium. For comparison, the EDDs of the beryllium that we obtained using a uniform prior distribution is shown in Fig. 5(b). The EDD shown in Fig. 5(b) should be the same as the ones shown by Iversen et al. [9]. Although the overall features are the same, there are some differences in the regions of low density. During the iterative process of maximization we first encountered some distributions that were rather similar to the ones obtained by Iversen et al. [9], but further maximization resulted in the EDD shown in Fig. 5(b).

The present study shows that it is absolutely necessary to include prior information in the MEM analysis when one wants to do accurate charge density studies. Maximizing the entropy using a uniform prior distribution can lead to seriously misleading artifacts such as non-nuclear maxima. In light of the results we have to conclude that there is no experimental evidence for the existence of nonnuclear maxima in $\mathrm{Si}$ and $\mathrm{Be}$.

[1] S. Besnainou, M. Roux, and R. Daudel, C.R. Acad. Sci. Paris 241, 311 (1955).

[2] W. L. Cao, C. Gatti, P. J. MacDougall, and R. F. W. Bader, Chem. Phys. Lett. 141, 380 (1987).

[3] G. I. Bersuker, C. Peng, and J.E. Boggs, J. Phys. Chem. 97, 9323 (1993).

[4] K.E. Edgecombe, V.H. Smith, and F. Müller-Plathe, Z. Naturforsch. 48a, 127 (1993).
[5] C. Mei, K. E. Edgecombe, V.H. Smith, and A. Heilingbrunner, Int. J. Quantum Chem. 48, 287 (1993).

[6] C. Pisani and R. Dovesi, Int. J. Quantum Chem. 17, 501 (1980).

[7] M. Sakata and M. Sato, Acta Crystallogr. Sect. A 46, 263 (1990).

[8] T. Saka and N. Kato, Acta Crystallogr. Sect. A 42, 469 (1986).

[9] B. B. Iversen, F. K. Larsen, M. Souhassou, and M. Takata, Acta Crystallogr. Sect. B 51, 580 (1995).

[10] F. K. Larsen and N. K. Hansen, Acta Crystallogr. Sect. B 40, 169 (1984).

[11] R. Y. de Vries, W. J. Briels, D. Feil, G. te Velde, and E. J. Baerends, Can. J. Chem. (to be published).

[12] H. Bruning, Ph.D. thesis, Univerity of Twente, The Netherlands (1992).

[13] E. T. Jaynes, Phys. Rev. 106, 620 (1957).

[14] E. T. Jaynes, Phys. Rev. 108, 171 (1957).

[15] S.F. Gull and G. J. Daniell, Nature (London) 272, 686 (1978).

[16] B. R. Frieden, J. Opt. Soc. Am. 62, 511 (1972).

[17] S. Kullback and R. A. Leibler, Ann. Math. Stat. 22, 79 (1951).

[18] R. Y. de Vries, W. J. Briels, and D. Feil, Acta Crystallogr. Sect. A 50, 383 (1994).

[19] J. Skilling and R. K. Bryan, Mon. Not. R. Astron. Soc. 211, 111 (1984).

[20] A. Zheludev, R. J. Papoular, E. Ressouche, and J. Schweizer, Acta Crystallogr. Sect. A 51, 450 (1995).

[21] G. te Velde and E. J. Baerends, Phys. Rev. B 44, 7888 (1991).

[22] G. J. M. Velders and D. Feil, Acta Crystallogr. Sect. B 45, 359 (1989).

[23] H. Bruning and D. Feil, Acta Crystallogr. Sect. A 48, 865 (1992). 